\title{
PÉRIODES DE NIVEAUX EXCITÉS DU CALCIUM 40 ET DE L'IODE 132
}

\author{
Par S. GORODETZKY, N. SCHULZ, \\ J. CHEVALLIER et A. C. KNIPPER, \\ Institut de Recherches Nucléaires, Strasbourg, France.
}

\begin{abstract}
Résumé. - Cet article présente la mesure de la période, $T_{1 / 2}=(2,15 \pm 0,08) \mathrm{ns} \mathrm{du}$ premier niveau excité de ${ }^{40} \mathrm{Ca}$. Les mesures de coïncidences différées dans ${ }^{132} \mathrm{I}$ ont permis de fixer l'ordre d'émission des deux rayons gamma de $52 \mathrm{keV}$ et $232 \mathrm{keV}$; les périodes sont égales à $T_{1 / 2}=(0,96 \pm 0,04) \mathrm{ns}$ pour le niveau à $52 \mathrm{keV}$ et à $T_{1 / 2}=(1,42 \pm 0,05) \mathrm{ns}$ pour le niveau à $284 \mathrm{keV}$. Ces valeurs, et des mesures du coefficient de conversion interne $\alpha_{K}$, permettent d'attribuer les ordres multipolaires $M 1+E 2$ et $E 2$ respectivement pour les transitions de 52 et $232 \mathrm{keV}$.
\end{abstract}

Abstract. - The half-life $T_{1 / 2}=(2.15 \pm 0.08) \mathrm{ns}$ has been measured for the first excited state in ${ }^{40} \mathrm{Ca}$. Delayed coincidence measurements in ${ }^{132} \mathrm{I}$ determine the emission order of the $52 \mathrm{keV}$ and $232 \mathrm{keV}$ gamma-rays ; half-lives have been measured as $T_{1 / 2}=(0.96 \pm 0.04)$ ns for the $52 \mathrm{keV}$ level and $T_{1 / 2}=(1.42 \pm 0.05) \mathrm{ns}$ for the $284 \mathrm{keV}$ level. These values, together with measured internal conversion coefficients $\alpha_{K}$, allow assignment of multipole orders $M 1+E 2$ and $E 2$ respectively for the $52 \mathrm{keV}$ and $232 \mathrm{keV}$ transitions.

Les mesures de vies moyennes décrites dans ce travail ont été effectuées à l'aide d'un montage lentrapide comprenant un convertisseur temps-amplitude basé sur le principe du chevauchement des impulsions [1]. Les pentes des courbes de coïncidences retardées obtenues avec des scintillateurs NE 102 couplés à des photomultiplicateurs 56 AVP, étaient analysées par la méthode des moindres carrés $\left({ }^{\mathbf{1}}\right)$.

I. Période du niveau excité à $3,35 \mathrm{MeV}$ du calcium 40. - I-1. Mesure. - Une cible de calcium naturel évaporé sur un support d'alumine était bombardée par un faisceau de protons à la résonance de 5,4 MeV [3]. Les électrons des paires internes par lesquelles le niveau à $3,35 \mathrm{MeV}$ de ${ }^{40} \mathrm{Ca}$ se désexcite étaient détectées par un scintillateur plastique de $4,5 \mathrm{~mm}$ d'épaisseur et de $10 \mathrm{~mm}$ de diamètre. Les feuilles d'aluminium interposées entre ce cristal et la cible correspondaient à $70 \mathrm{mg} / \mathrm{cm}^{2}$ d'absorbant, c'est-à-dire à une perte d'énergie de $0,3 \mathrm{MeV}$ pour les électrons et de $6 \mathrm{MeV}$ pour les protons. Ceux-ci étaient détectés dans un scintillateur NE 102 de $0,2 \mathrm{~mm}$ d'épaisseur couplé directement (sans graisse de silicone) à la photocathode du photomultiplicateur et maintenu par une feuille de $0,005 \mathrm{~mm}$ d'aluminium. La sélection d'énergie était placée sur le pic $p_{1}^{\prime}$ des protons conduisant au premier état excité (fig. 1).

(1) Les valeurs des trois mesures de vies moyennes ont été reportées, comme résultats préliminaires, dans un résumé des vies moyennes mesurées à l'Institut de Recherches Nucléaires de Strasbourg [2].

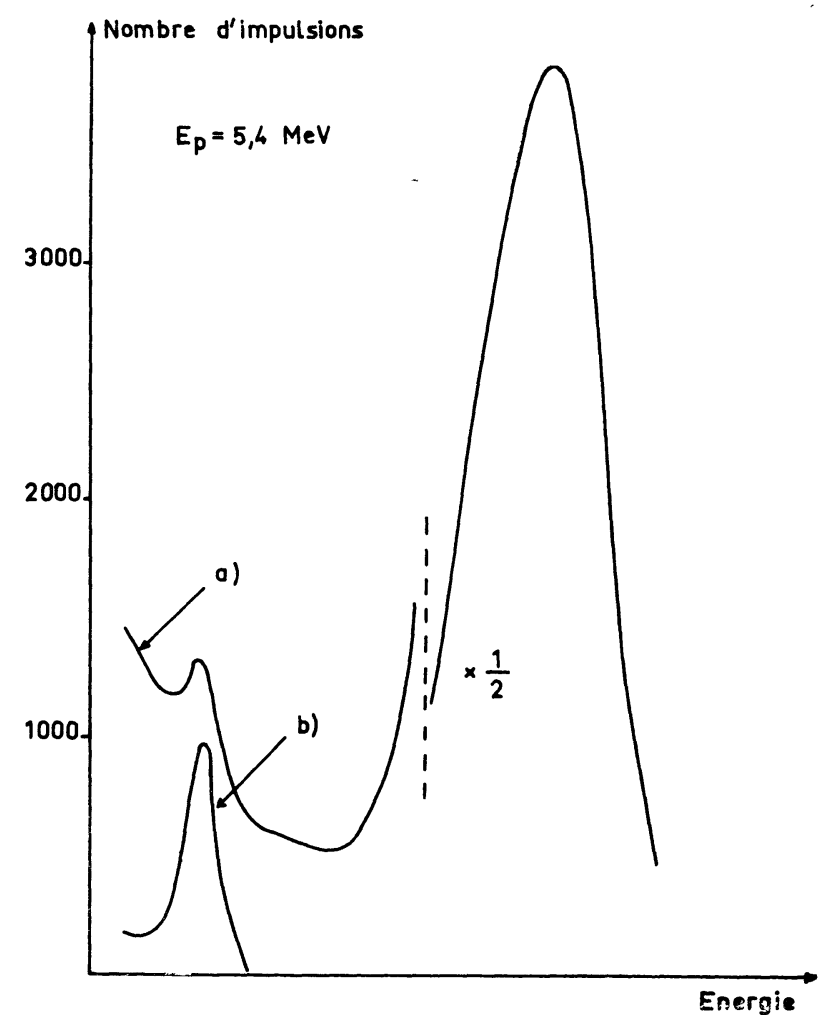

FIg. 1. - Réaction ${ }^{40} \mathrm{Ca}\left(\mathrm{pp}^{\prime}\right)$. Courbe $a$ : spectre des protons. Courbe $b$ : même spectre en cö̈ncidence avec les électrons des paires internes.

La figure 2 montre l'une des deux courbes de coïncidences retardées obtenue. Une prompte a été mesurée en coïncidences $p_{3}^{\prime}-\gamma$ dans la réaction 
${ }^{27} \mathrm{Al}\left(\mathrm{pp}^{\prime}\right)$; l'énergie de bombardement des protons était choisie de façon à faire coöncider l'énergie des protons $p_{3}^{\prime}$ avec celle des protons $p_{1}^{\prime}$ de la réaction ${ }^{40} \mathrm{Ca}\left(\mathrm{pp}^{\prime}\right)$. Quoique n'étant pas obtenue dans les mêmes conditions, les électrons ayant été remplacés par des rayons gamma, cette prompte indique que la pente de la courbe de résolution est environ quatre fois plus forte que celle des courbes de coïncidences retardées. L'analyse de ces dernières conduit à la valeur

$$
T_{1 / 2}=(2,15 \pm 0,08) \mathrm{ns}
$$

pour la période du niveau à $3,35 \mathrm{MeV}$ de ${ }^{40} \mathrm{Ca}$.

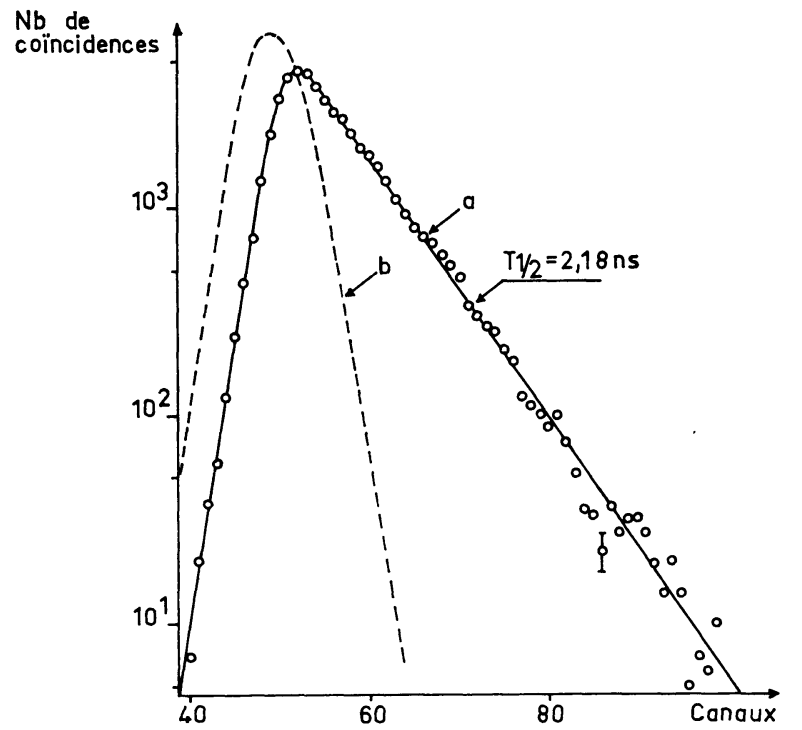

Fig. 2. - Période du premier niveau excité de ${ }^{40} \mathrm{Ca}$.

Courbe $a$ : courbe de coïncidences retardées $\mathrm{p}_{1}^{\prime}-\mathrm{e}$ obtenue dans la réaction ${ }^{40} \mathrm{Ca}\left(\mathrm{pp}^{\prime}\right)$. Courbe $b:$ courbe de coïncidences promptes $\mathrm{p}_{\boldsymbol{\beta}}^{\prime}-\gamma$ obtenue dans la réaction $\mathrm{Al}^{27}\left(\mathrm{pp}^{\prime}\right)$.

Cette vie moyenne a aussi été déterminée par Kloepper, Day et Lind [4] en observant à l'aide d'un compteur à scintillations $\mathrm{NaI}(\mathrm{Tl})$ la décroissance en fonction du temps de l'état produit par des faisceaux pulsés de neutrons et de protons. La valeur publiée $\tau=(3,4 \pm 0,2)$ ns est la combinaison des résultats correspondants aux réactions

$$
{ }^{40} \mathrm{Ca}\left(\mathrm{nn}^{\prime}\right): \tau=(3,6 \pm 0,2) \mathrm{ns}
$$

et

$$
{ }^{40} \mathrm{Ca}\left(\mathrm{pp}^{\prime}\right): \tau=(3,2 \pm 0,2) \mathrm{ns} .
$$

Cette dernière valeur est en bon accord avec notre résultat : $\tau=(3,10 \pm 0,12) \mathrm{ns}$.

I-2. Discussion. - L'élément de matrice monopolaire $M=\left\langle f\left|\sum_{p} r_{p}^{2}\right| i\right\rangle$ de transition des processus à électrons peut être mis sous la forme $M=\rho R^{2}$ où $R$ est le rayon du noyau et où le para- mètre $\rho$ caractérise l'intensité de l'interaction nucléaire. Ce paramètre est déterminé par la relation $\rho^{2}=T_{i} / F_{i}, T_{i}$ étant la probabilité de transition et $F_{i}$ une fonction [5] du nombre atomique et de l'énergie de la transition $(i=e$ ou $\pi$ suivant que la transition a lieu par conversion interne ou par émission de paires internes).

Dans ${ }^{40} \mathrm{Ca}$, l'intensité de la désexcitation du premier niveau par conversion interne n'est que de $0,64 \%$ comparée à l'émission de paires internes [6]. La valeur expérimentale de $\rho$ déduite de cette dernière est de 0,15 . Alors qu'une valeur théorique, basée sur l'excitation d'une particule, donne 0,8 [7], un calcul faisant intervenir un mélange de configurations à une et à deux particules conduit à la valeur de 0,26 [8]. Pour la transition monopolaire de ${ }^{16} \mathrm{O}$, noyau plus étudié que ${ }^{40} \mathrm{Ca}$, on constate aussi que les excitations de plusieurs particules rendent mieux compte de la valeur expérimentale de $\rho$ qu'une excitation d'une particule; quant aux modèles collectifs, ils conduisent à des valeurs de $\rho$ encore plus fortes que celle donnée par l'excitation d'une particule.

II. Périodes de niveaux excités de l'iode 132. Une cascade de deux rayons gamma de 52 et $232 \mathrm{keV}$ suit la désintégration bêta $(220 \mathrm{keV})$ de ${ }^{132} \mathrm{Te}$ (78 heures). Les multipolarités proposées par Cheever et coll. [9] pour ces deux transitions dans 132I ont été confirmées par la mesure des coefficients de conversion interne $\alpha_{K}$. Les courbes de coïncidences retardées $\gamma-X$ et $\beta-\gamma$ ont permis de fixer l'ordre d'émission des deux rayons gamma (fig. 3).

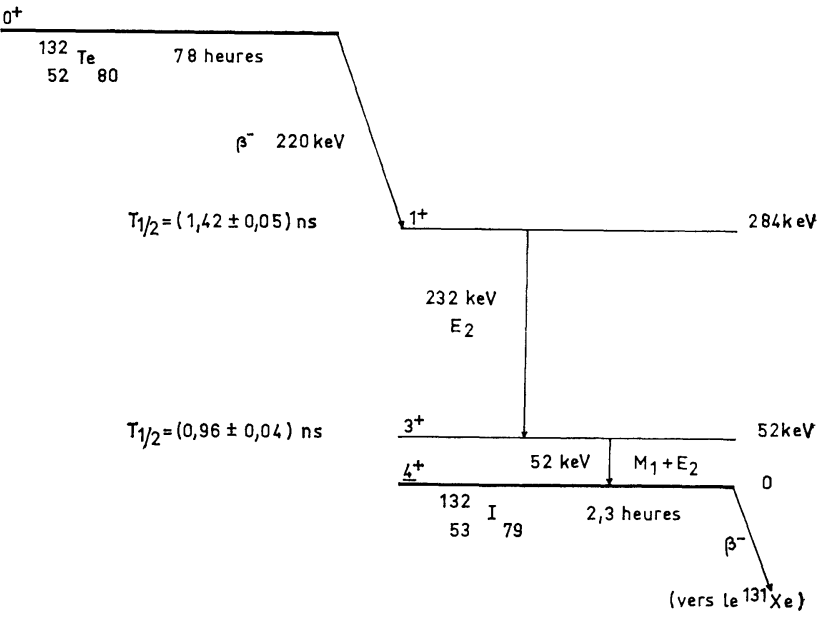

Fig. 3. - Schéma de désintégration du tellure 132. Un niveau $J^{\pi}=2^{+}$à $161,48 \mathrm{keV}$ (ou à 166,02 keV) a été signalé par Fransson et Bemis [11].

II-1. Mesures de Spectroscopie nuCléaire. Une étude spectroscopique des niveaux d'énergie de ${ }^{132}$ I est rendue difficile par la décroissance 
de ${ }^{132} \mathrm{I}$ (2,3 heures) vers ${ }^{132} \mathrm{Xe}:$ des spectres bêta allant jusqu'à $2,1 \mathrm{MeV}$ et gamma jusqu'à $2,2 \mathrm{MeV}$ masquent les transitions à étudier. De ce fait, les sources liquides de ${ }^{132} \mathrm{Te}$ obtenues du RCC Amersham ou de Saclay, étaient épurées immédiatement avant leur utilisation et déposition sous forme solide. L'isotope ${ }^{132}$ I était entraîné par des vapeurs d'iode inactif rajouté à la solution.

Avec ces sources de ${ }^{132} \mathrm{Te}$ traitées, nous avons déterminé les énergies des rayons gamma, en utilisant un cristal $\mathrm{NaI}$ calibré à l'aide des sources de ${ }^{57} \mathrm{Co},{ }^{111} \mathrm{Ag},{ }^{131} \mathrm{I},{ }^{166} \mathrm{Ho},{ }^{170} \mathrm{Tm}$ et ${ }^{203} \mathrm{Hg}$. Des mesures du spectre gamma en coïncidence avec l'une ou l'autre des radiations nous ont fourni les coefficients $\alpha_{\mathrm{K}}$; les résultats sont reportés dans le tableau I en même temps que les mesur'es d'autres auteurs. La comparaison des coefficients $\alpha_{K}$ expérimentaux et théoriques montre que la transition de $52 \mathrm{keV}$ est une transition $M_{1}+E_{2}$ où la composante dipolaire magnétique prédomine. D'après le schéma de désintégration, la transition de $232 \mathrm{keV}$ est alors nécessairement quadrupolaire électrique, ce qui est confirmé tant par les mesures de coefficients de conversion interne $\left(\alpha_{K}\right.$ : voir tableau I, rapports $L_{\mathrm{I}} / L_{\mathrm{II}} / L_{\mathrm{III}}$ : [11]) que par la mesure de vie moyenne.

Des mesures d'activité du ${ }^{132} \mathrm{Te}$ avec son descendant ${ }^{132} \mathrm{I}$ en équilibre ont montré qu'il y a $0,98 \pm 0,10$ rayons gamma d'énergie $232 \mathrm{keV}$ par désintégration; la radioactivité bêta de ${ }^{132} \mathrm{Te}$ n'a donc pas d'embranchement. Dans un travail récent, Fransson et Bemis [11] ont relevé l'existence de deux rayons gamma de 112 et $116 \mathrm{keV}$ en cascade entre les niveaux à 284 et à $52 \mathrm{keV}$; leur intensité est de $2 \%$ par rapport à la transition de $232 \mathrm{keV}$.

II-2. Mesures de temps de vie. - La figure 4 montre le spectre gamma d'une source épurée de ${ }^{132} \mathrm{Te}$ dans un scintillateur plastique tronconique $(\phi=20 \mathrm{~mm}$ et $12 \mathrm{~mm}, h=20 \mathrm{~mm})$. Le pic observé à $28 \mathrm{keV}$ (l'énergie Compton du rayon gamma de $122 \mathrm{keV}$ de ${ }^{57} \mathrm{Co}$ est de $39,5 \mathrm{keV}$ ) est le pic photoélectrique des rayons $\mathrm{X}$ de l'iode provenant de la conversion interne des deux rayons gamma. Une première série de mesures de temps a été réalisé en

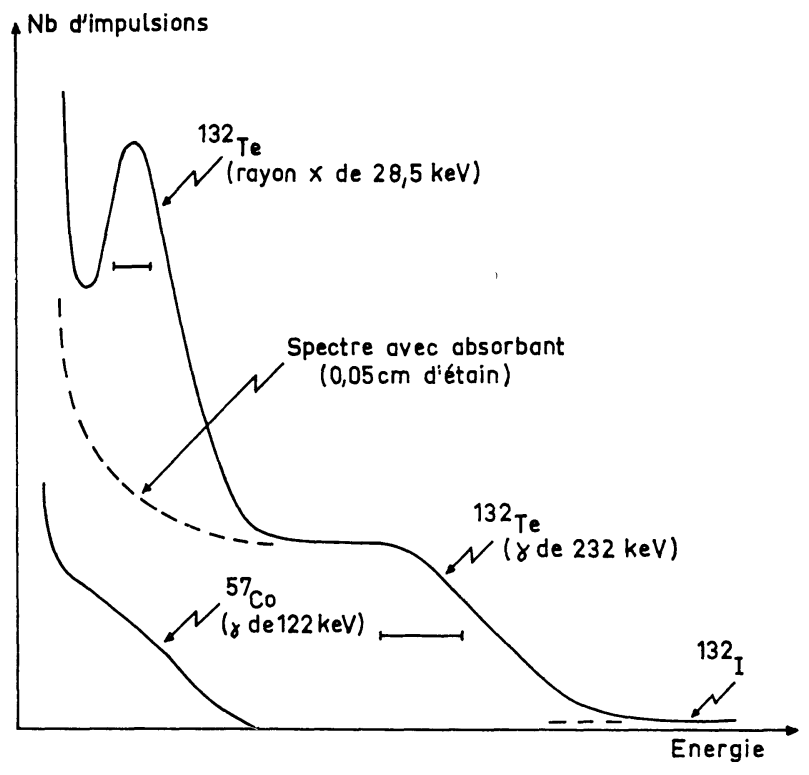

Fig. 4. - Spectre du rayonnement gamma d'une source épurée de tellure 132 (scintillateur plastique NE102) et sélections d'énergie.

coïncidences $\gamma-X$ avec les sélections d'énergie indiquées à la figure 4. Un exemple de ces mesures est illustré par la figure 5 qui permet de constater que le rayon gamma de $230 \mathrm{keV}$ est la radiation mère. Une courbe de résolution prompte en ${ }^{22} \mathrm{Na}$, obtenue avec les mêmes sélections d'énergie, indique une pente à droite de $0,44 \mathrm{~ns}$. Le résultats des différentes mesures conduit à la valeur de

$$
T_{1 / 2}=(0,96 \pm 0,04) \mathrm{ns}
$$

pour la période du niveau excité à $52 \mathrm{keV}$. Aucune correction n'est à faire pour le retard introduit par l'émission $\mathrm{X}$ puisque la largeur du niveau $K$ est de $10 \mathrm{eV}$ [13].

Pour la détection des rayons bêta, l'un des deux cristaux tronconiques a été remplacé par un cristal cylindrique de $15 \mathrm{~mm}$ de diamètre. L'épaisseur, $e=5 \mathrm{~mm}$, a été choisie pour pouvoir, dans les sélections d'énergie, éviter le plus possible la contribution des rayons $\beta$ de grande énergie provenant

TABLEAU I

\begin{tabular}{|c|c|c|c|c|c|}
\hline & \multicolumn{3}{|c|}{$\gamma_{1}$} & \multicolumn{2}{|c|}{$\gamma_{2}$} \\
\hline & $E(\mathrm{keV})$ & & $\alpha_{K}$ & $E(\mathrm{keV})$ & $\alpha_{K}$ \\
\hline Cheever et coll. [9] & $(230)$ & 0,05 & $\pm 0,04$ & $53 \pm 5$ & $5,35 \pm 0,22$ \\
\hline $\begin{array}{l}\text { Ce travail } \\
\text { Iyanov et coll. }[10]\end{array}$ & $232 \pm 3$ & 0,087 & 士: 0,022 & $52 \pm 2$ & 5,4 \\
\hline $\begin{array}{l}\text { Ivanov et coll. }[10] \\
\text { Fransson et Bemis }[11]\end{array}$ & $22816+006$ & 0,06 & $\pm 0,01$ & $4972+001$ & $1,00 \pm 0,50$ \\
\hline $\begin{array}{l}\text { Fransson et Bemis [11] } \\
\text { Valeurs théoriques [12] }\end{array}$ & $228,10 \pm 0,00$ & $\begin{array}{r}0,076 \\
M \\
E_{2}\end{array}$ & $\begin{array}{l}1: 0,067 \\
:: 0,081\end{array}$ & 49,12 士 0,01 & $\begin{array}{c}4,94 \pm 0,00 \\
M_{1}: 4,9 \\
E_{2}: 8,2\end{array}$ \\
\hline
\end{tabular}

Énergies et coefficients de conversion interne $\alpha_{K}$ des deux transitions gamma observées dans l'iode 132. Les valeurs théoriques des coefficients $\alpha_{K}$ correspondent aux énergies déterminées par Fransson et Bemis [11]. 


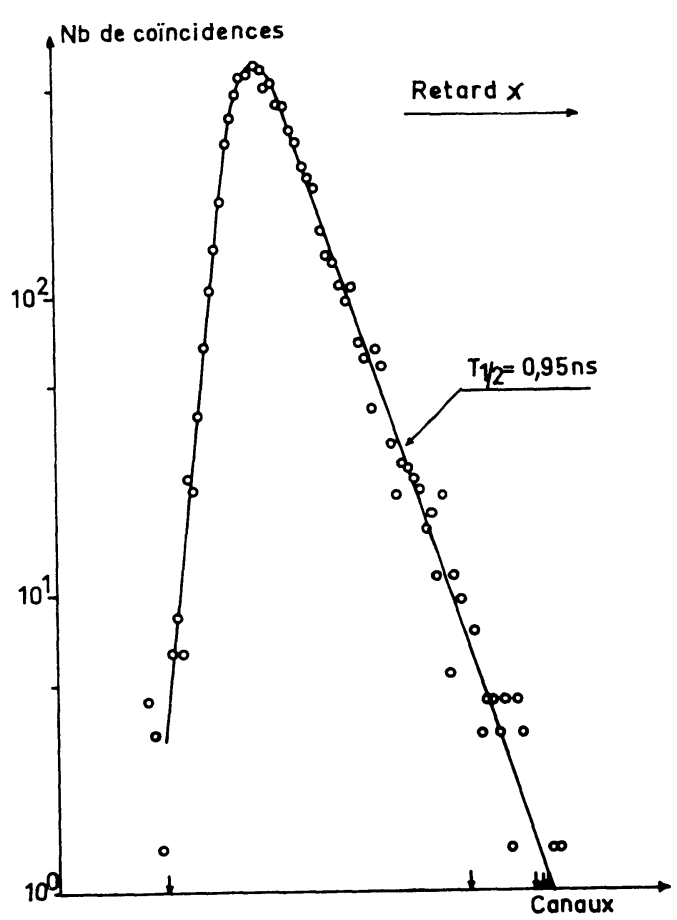

FIG. 5. - Période du niveau excité à $52 \mathrm{keV}$ de l'iode 132. Courbe de coïncidences retardées $\beta 232 \mathrm{keV}-\mathrm{X}$.

de la désintégration de ${ }^{132} \mathrm{I}$ qui se reforme dans les sources épurées. Cette contribution est visible dans le spectre de temps des coïncidences $\beta--\gamma$ (fig. 6). La moyenne pondérée de nos différentes mesures indique une période

$$
T_{1 / 2}=(1,42 \pm 0,05) \mathrm{ns}
$$

pour le niveau excité à $284 \mathrm{keV}$.

Nous avons procédé à une mesure de contrôle en enregistrant la courbe de coïncidences retardées $\beta--\mathrm{X}$. Cette courbe $F(t)$ est la somme d'une prompte $P(t)$ et de deux courbes de coïncidences retardées, l'une correspondant à la vie moyenne $\tau_{1}=1 / \lambda_{1}$ du niveau à $284 \mathrm{keV}$ et l'autre aux deux vies moyennes $\tau_{1}$ et $\tau_{2}=1 / \lambda_{2}$ en cascade :

$$
\begin{aligned}
F(t)=\alpha P(t)+ & \beta \lambda_{1} \int_{-\infty}^{+\infty} \mathrm{e}^{-\lambda_{1} \mathbf{T}} P(t-T) \mathrm{d} T \\
& +(1-\alpha-\beta) \lambda_{1} \lambda_{2}\left(\lambda_{2}-\lambda_{1}\right)^{-1} \\
& \int_{-\infty}^{+\infty}\left(\mathrm{e}^{-\lambda_{1}^{\prime} \mathrm{T}}-\mathrm{e}^{-\lambda_{2}^{i} \mathrm{~T}}\right) P(t-T) \mathrm{d} T .
\end{aligned}
$$

Lorsque $t$ croît, la courbe $\log F(t)$ tend vers une droite de pente $-\lambda_{1}$ (fig. 7).

Discussion. - Le spin mesuré 4 de l'état fondamental est en accord avec la règle de couplage faible de Nordheim [14] si le proton impair se trouve dans l'état $g_{7 / 2}$ et le neutron dans l'état $d_{3 / 2}$ ainsi que le

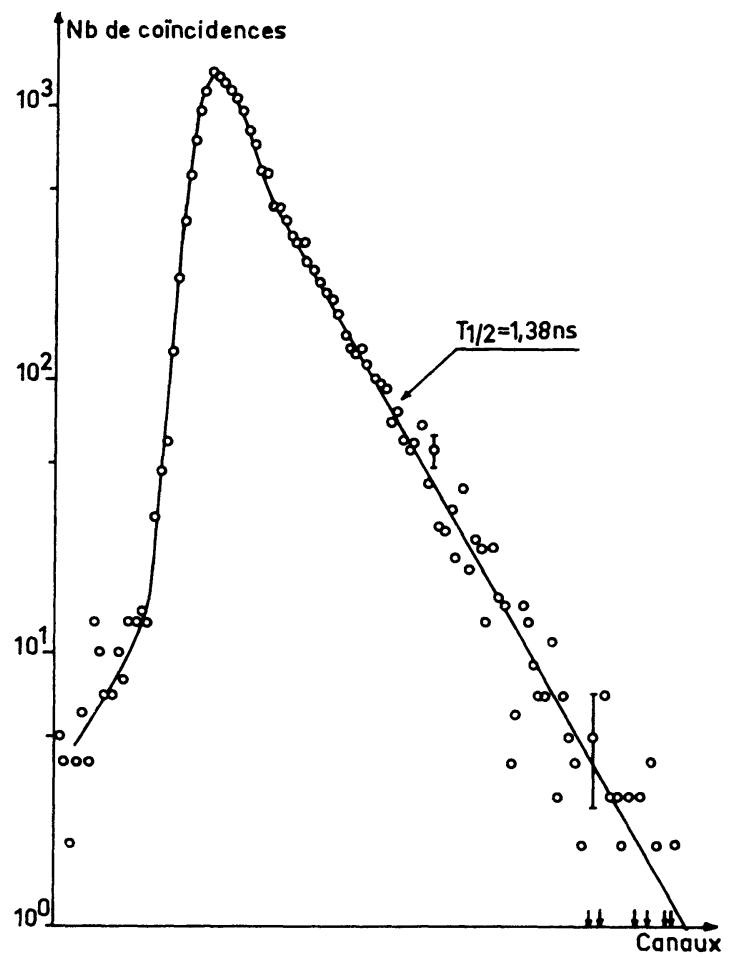

Fig. 6. - Période du niveau excité à $284 \mathrm{keV}$ de l'iode 132. Courbe de coïncidences retardées $\beta-\gamma 232 \mathrm{keV}$.

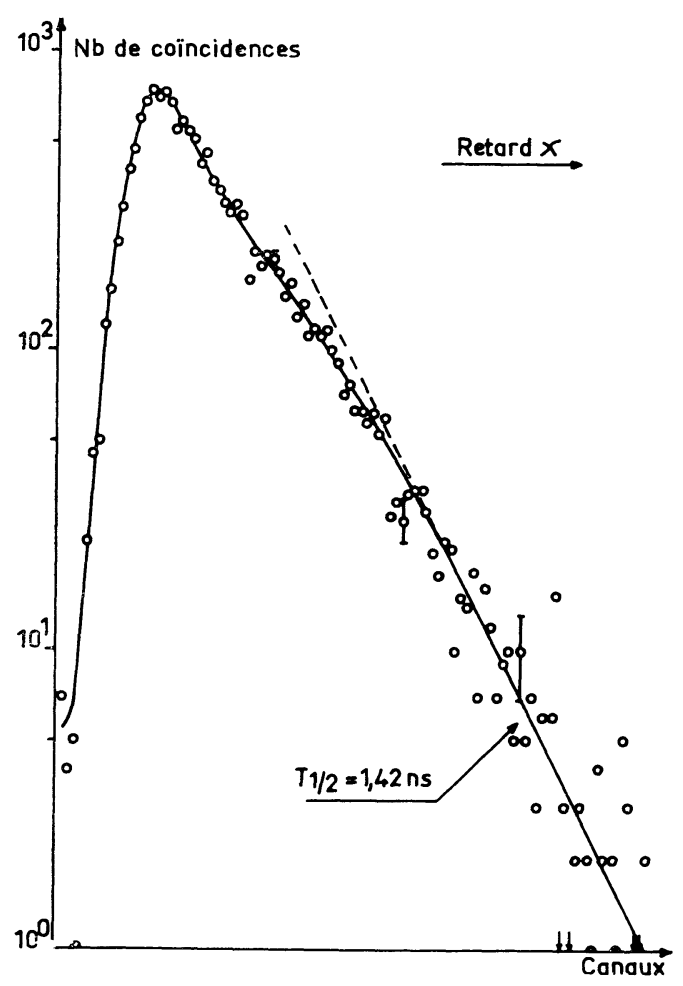

Fig. 7. - Courbe de coïncidences différés $\beta-\mathrm{X}$ (source de tellure 132 épurée). 
prévoit la séquence de niveaux proposés par Klinkenberg [15].

L'énergie d'excitation du premier niveau est de $52 \mathrm{keV}$, comme le montrent nos mesures. Cette transition peut correspondre :

- soit à une transition neutronique $s_{1 / 2} \rightarrow d_{3 / 2}$;

- soit à une transition protonique entre les états $g_{7 / 2}$ et $d_{5 / 2}$ comme dans les noyaux voisins ${ }^{125} \mathrm{I}$, ${ }^{127}$ I, ${ }^{125}$ I et ${ }^{125}$ I [16] ;

- soit à une transition entre deux états de la même configuration $\left(g_{7 / 2}\right)^{3}\left(d_{3 / 2}\right)^{1}$.

Dans ces trois cas, la composante dipolaire magnétique de la transition doit être ralentie. Le facteur de ralentissement est de l'ordre de 60 pour une transition neutronique $M_{1} l$ interdite dans les noyaux impairs [17], de 100 à 200 pour les transitions $M_{1} l$ - interdites dans les isotopes impairs de l'iode [16] et de 30 à 300 pour les transitions entre états de même configuration [18]. En tenant compte du coefficient de conversion interne $\alpha_{K}$, du coefficient $\delta^{2}=2,5 \times 10^{-4}[11]$ et du rapport d'embranchement, l'on déduit de la période du premier niveau excité un facteur de ralentissement de 35 pour la composante $M_{1}$ de la transition. Les connaissances actuelles concernant le noyau impair - impair ${ }^{132}$ I ne nous permettent pas de nous prononcer en faveur de l'une des configurations proposées pour le premier niveau.

La vie moyenne partielle $\tau_{\gamma}=2,2 \mathrm{~ns} \mathrm{du}$ niveau excité à $284 \mathrm{keV}$ renforce l'hypothèse que la transition de $232 \mathrm{keV}$ est une transition quadrupolaire électrique ; en effet, la formule de Weisskopf, indique une vie moyenne de $30 \mathrm{~ns}$ si le niveau se désexcite par une transition $E_{2}$.

Remerciements. - Nous tenons à remercier MM. K. Fransson et C. E. Bemis de nous avoir communiqué les résultats de leurs travaux avant publication.

Manuscrit reçu le 1er avril 1966.

\section{BIBLIOGRAPHIE}

[1] Gorodetzky (S.), Richert (R.), Manquenouille (R.) et Knipper (A.), Nucl. Instr. and Meth., 1960, 7, 50.

[2] Gorodetzky (S.) et coll., Proc. Gatlingburgh Conf. on Electromagnetic Lifetimes and Properties of Nuclear States, p 79 N A S, DC 1962.

[3] Bent (R. D.) et Kruse (T. H.), Phys. Rev., 1958, $109,1240$.

[4] Kloepper (R. M.), Day (R. B.) et Lind (D. A.), Phys. Rev., 1959, 114, 240.

[5] Thomas (R.), Phys. Rev., 1940, 58, 714.

[6] Nessin (M.), Eklund (K. E.) et Kruse (T. H.), B. A. P. S., 1959, 4, 278.

[7] Berenbaum (R.) et Umezawa (M.), Nuclear Physics 1962, 39, 177.

[8] Visscher (W. M.), Communication privée citée par Kloepper et coll. [4].

[9] Cheever (G. D.), Koski (W. S.), Tilley (D. R.) et Madansky (L.), Phys. Rev., 1958, 110, 922 .
[10] Yvanov (Y. F.), Rumer (I. A.) et Bukack (A. Y.), Izv. Akad. Nauk. SSSR, Ser. Fiz., 1965, 29, 157.

[11] Fransson (K.) et Bemis (C. E.), Nuclear Physics, 1966, 78, 207.

[12] Surv (L. A.) et Band (I. M.), Tables of Internal Conversion Coefficients, 1961.

[13] Callan (E. J.), Conference on the Role of Atomic Electrons in Nuclear Transformations, Varsovie, 1963.

[14] Nordheim (L. W.), Rev. Mod. Physics, 1951, 23, 322.

[15] Klinkenberg (P. F. A.), Rev. Mod. Physics, 1952, 24, 63.

[16] Bemis (C. E.) et Fransson (K.), Phys. Letters, 1965, 19, 567 .

[17] Way (K.), Kundu (D. N.), McGinnis (C. L.) et Van Lieshout (R.), Ann. Rev. Nucl. Sc., 1957, 6, 129.

[18] Talmi (I.)”et Unna (I.), Ann. Rev. Nucl. Sc., 1960, 10,353 . 